\title{
Distinguishing Among Models of Contextually Induced Preference Reversals
}

\author{
Douglas H. Wedell \\ University of South Carolina
}

\begin{abstract}
Previous research has demonstrated that adding a 3rd (decoy) alternative that is dominated by only 1 of the other 2 alternatives in a choice set increases the preference for the dominating alternative (J. Huber, Payne, \& Puto, 1982; Tyszka, 1983). By manipulating decoys within subjects, significant preference reversals have been obtained. The processes of 3 classes of models can account for these reversals: (a) The decoy affects the weights assigned to different dimensions, (b) the decoy produces range-frequency effects on the dimensional values of the alternatives, and (c) the perception of dominance directly increases the attractiveness of the targeted alternative. The results of 3 experiments designed to test these models favored a direct effect of dominance. When a dominated decoy was added to a choice set, asymmetrical dominance was demonstrated to be a necessary and sufficient condition for producing the predicted preference reversals.
\end{abstract}

Preference reversals have intrigued decision scientists in much the same way that visual illusions have captured the attention of researchers investigating visual perception. In each case, the respective cognitive system appears to operate reasonably well in the normal environment. Yet, when brought into a laboratory situation in which stimulus information can be selectively manipulated, the systems can be made to break down, resulting in behaviors (or perceptions) that sometimes defy common sense. If one focuses only on these results, it is tempting to conclude that the cognitive system is poorly designed. However, the functional adequacy of the system under normal conditions implies that this is not the case. Instead, these systemic breakdowns provide theoretical leverage for better understanding the processes that govern decision making under normal conditions.

Three broad classes of preference reversals have been reported in the literature. By far, the most extensively researched of these is the task-induced preference reversal, in which preference ordering is demonstrated to differ depending on the mode of response required by the task. Much of this research has involved comparison of how gambles are evaluated (Casey, in press; Goldstein \& Einhorn, 1986; Grether \& Plott, 1979; Lichtenstein \& Slovic, 1971, 1973; Lindman, 1971; Slovic \& Lichtenstein, 1983; Wedell \& Böckenholt, 1990), although recently this work has been extended to evaluations of nonprobabilistic alternatives (Tversky, Sattath, \& Slovic, 1988). A generally accepted explanation of taskinduced preference reversal is that the response mode affects how different dimensions are weighted. For example, probability information is generally given greater weight when preferences for gambles are expressed through choice rather than through pricing procedures. Various explanations of why

This article benefited from the helpful comments of Gary Allen, Ulf Böckenholt, Mike Brondino, Alan Lambert, and three anonymous reviewers.

Correspondence concerning this article should be addressed to Douglas H. Wedell, Department of Psychology, University of South Carolina, Columbia, South Carolina 29208. response mode affects weighting include considerations of the more lexicographic nature of choice rather than matching procedures, the ease with which different types of information are translated to different response formats, and the role of the decision maker's aspiration level in choice and judgment.

A second, widely reported class of preference reversals may be described as frame induced. In these situations, subjects evaluate problems that differ in their surface structure but share the same basic deep structure (e.g., reporting of lives lost versus lives saved). Strong reversals of preference ordering have been demonstrated to result from different frames of the same problem (Fischhoff, Slovic, \& Lichtenstein, 1980; Kahneman \& Tversky, 1979, 1984; Slovic, Fischhoff, \& Lichtenstein, 1982; Tversky \& Kahneman, 1981), although the stability and predictability of framing effects across problem domains have been challenged (Fischhoff, 1983; Hershey \& Schoemaker, 1980; Schneider \& Lopes, 1986). According to prospect theory (Kahneman \& Tversky, 1979), the general explanation of framing-induced preference reversals is twofold. First, it is assumed that decision makers tend to operate on an edited rather than full version of the decision problem; the different frames then represent different edited versions of the same problem. Second, valuation of the alternatives differs across different frames. For example, the utility function for losses is proposed to be steep and convex, whereas the function for gains is proposed to be more gradual and concave.

A third type of preference reversal may result from manipulation of the set of (contextual) stimuli included for evaluation or choice. This article investigates one type of contextually induced reversal, resulting from the introduction of an asymmetrically dominated alternative into the choice set. An asymmetrically dominated alternative is one that is dominated by at least one of the choice alternatives in the set but is not dominated by another of the alternatives. ${ }^{1}$ Tyszka

\footnotetext{
' Alternative $A$ dominates alternative $B$ if for every attribute of $B$, the value of that attribute for $A$ is greater than or equal to the value for $B$ and if for at least one attribute, the value for $A$ is strictly greater than the value for $B$.
} 
(1983) demonstrated that adding such an alternative (a decoy) to the choice set increases the probability of choosing the alternative that dominates the decoy. J. Huber, Payne, and Puto (1982) further demonstrated that by presenting the choice pair twice and varying which alternative dominates the decoy, one can cause statistically significant reversals of preference to occur. Several subsequent studies replicated this effect (Ratneshwar, Shocker, \& Stewart, 1987; Simonson, 1989).

J. Huber and Puto (1983) classified the asymmetrical dominance effect under the broader category of "attraction" effects, which can occur even when the decoy is not dominated by either of the choice alternatives. Although nondominated and asymmetrically dominated decoys can have similar effects on choice, the processes underlying these effects may not be the same. The experiments reported here are limited to situations in which the decoy is dominated by at least one of the other choice alternatives. Results from research on nondominated decoys are discussed when they are relevant to the three models described below. A fuller discussion of these effects and how they relate to the results reported here is given in the General Discussion section.

\section{Three Models of Contextually Induced Preference Reversals}

As J. Huber et al. (1982) pointed out, several explanations are possible for contextually induced preference reversals resulting from the manipulation of dominance relations between the decoy and the targeted set. The experiments reported here were designed to test three broad conceptualizations of the asymmetrical dominance effect as depicted in the panels of Figure 1. In each panel, two choice alternatives, $A$ and $B$, are located in a two-dimensional space. The direction of the preference vector along the diagonal indicates that the two dimensions are initially weighted equally and that the two choice alternatives lie on the same equi-preference contour. The panels indicate how different decoys can increase preference for alternative $A$ through theoretical mechanisms that are described in detail below.

\section{Dimensional Weight Model}

According to this model, a decoy can affect choice by increasing the relative weight given to one of the dimensions (depicted as a rotation of the preference vector through the stimulus space) so that alternatives $A$ and $B$ lie on different equi-preference contours. This is a very general model that has been used by Tversky et al. (1988) and others to explain task-induced preference reversals.

Clearly, for this type of model to apply, dimensional weights must to some extent depend on the values of the contextual stimuli being considered. Thus, this model is an instance of what are broadly referred to as differential-weighting models (Anderson, 1981). Research on these models has demonstrated that differential weights may depend on, among other things, the salience of the information (Fiske, 1980), the diagnosticity of the information (Skowronski \& Carlston, 1989 ), and the reliability of the information (Birnbaum \& Stegner, 1979).

For the dimensional weight model to make testable predictions of when preference reversals will occur, a relationship must be specified between the location of the decoy in the multidimensional space and the weight accorded to each dimension. The decoy depicted in the first panel of Figure 1, $R_{A}$, extends the range of variation along Dimension I but not along Dimension II. This manipulation has been demonstrated to increase preference for Target $A$ (J. Huber et al., 1982), which can be interpreted within the dimensional weight model as resulting from a decrease in the relative weight accorded Dimension I.

Why should extending the range along a dimension decrease the relative weight given to that dimension? One possible explanation is to conceive of the judgment process in terms of an additive difference model (Tversky, 1969). This type of model corresponds to dimensionwise rather than alternativewise processing of information, in which differences along each dimension are weighted and summed. From this perspective, it is perhaps not too surprising that the same difference in values may receive less relative weight when that difference is small in comparison with the range of possible differences implied by the contextual set than when it is large in comparison with that range. Consistent with this idea is

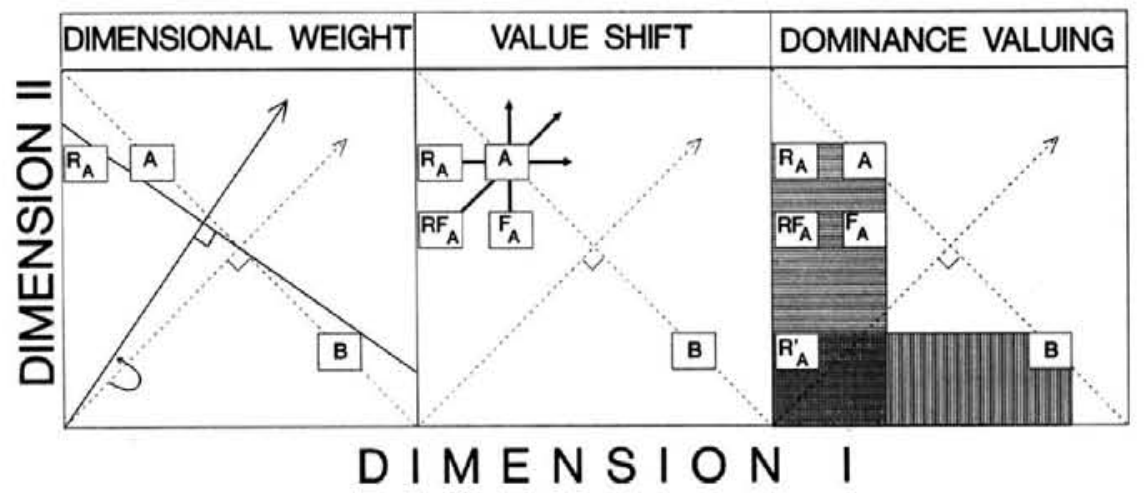

Figure 1. Three models of the effects of decoys on preference for target alternatives (see text for details). 
work in psychophysical judgment that has demonstrated that the same difference is more discriminable when the range of contextual variation along that dimension is small rather than large (Braida \& Durlach, 1972; Gravetter \& Lockhead, 1973; Parducci \& Perrett, 1971).

Thus, a testable version of the dimensional weight model is that the weight accorded a dimension is inversely related to the range of variation along that dimension. This may be thought of in terms of the same difference along a dimension being accorded more attention, or being viewed as more important or reliable, when it is large in relation to the range of contextual variation along that dimension. Implications of this model are discussed in relation to the implications of the other two models.

\section{Value Shift Model}

The middle panel of Figure 1 depicts the value shift model. According to this model, changes in preferences caused by inclusion of the decoy do not result from a change in the relative weighting of the dimensions but rather from a shift in the subjective values of the stimuli along the dimensions of judgment. Thus, like explanations of frame-induced preference reversals, the valuation of the alternatives is assumed to change across contexts.

A vast literature describes the effects of manipulating the contextual set of stimuli on unidimensional judgments (for reviews, see Eiser \& Stroebe, 1972; Helson, 1964; Parducci, 1983; Wedell, 1990). For judgments along a wide spectrum of dimensions, contextual contrast is generally observed: The judged value of the stimulus is displaced away from the values of contextual stimuli. The three decoys depicted in the middle panel of Figure 1 are derived from Parducci's (1965) rangefrequency theory, which has been demonstrated to provide good quantitative predictions of the effects of manipulating the contextual distribution in both psychophysical and social judgment domains (e.g., Birnbaum, 1974; Mellers, 1982; Parducci \& Wedell, 1986; Riskey, Parducci, \& Beauchamp, 1979; Wedell, Parducci, \& Lane, 1990; Wedell, Parducci, \& Roman, 1989). According to the theory, the subjective value of the stimulus reflects (a) its position in relation to the minimum and maximum values considered along the dimension of judgment (i.e., its range value) and (b) the relative frequency of stimuli lying below it (i.e., its frequency value).

Thus, insofar as $R_{A}$ extends the subjective range along Dimension I, its inclusion in the choice set should increase the subjective value of alternative $A$ along Dimension I (indicated by an arrow in Figure 1). However, because $B$ already lies at or near the maximum value along Dimension I, the range decoy should have little effect on the judged value of $B$. The result of including $R_{A}$ in the choice set then is to displace the location of alternative $A$ onto a new equi-preference contour, increasing its choice probability in relation to alternative $B$. Similarly, inclusion of the frequency decoy $\left(F_{A}\right)$ should increase the proportion of stimuli lying below alternative $A$ along Dimension II and hence increase its subjective value along that dimension. The effect of the $F_{A}$ decoy on alternative $B$ will be in the opposite direction. Thus, once again the preference for $A$ should be enhanced. Finally, both range and frequency effects should operate on the dimensional values of alternative $A$ when the range-frequency decoy $\left(R F_{A}\right)$ is included in the choice set.

Some support for the value shift model is provided by Study 3 of Ratneshwar et al. (1987), in which subjects made ratings of the quality of products in choice sets composed of a decoy (either asymmetrically dominated or nondominated) and two other alternatives. Information for all product domains varied on two dimensions: price and a quality-related attribute. Consistent with a value shift interpretation, quality ratings of an alternative were significantly higher when the choice set included a decoy designed to enhance preference for that alternative than when the decoy favored the other alternative. However, this result in itself does not imply that the decoy's effect on choice is guided by a shift in attribute values. Clearly, failure to find such a difference in dimensional judgments would be problematic for the value shift model, but the observed shift may be unique to the judgment situationwith different processes involved in choice. Alternatively, the shift in value may itself result from an implicit choice process in which the targeted alternative is preferred because of other mechanisms (such as dimensional weighting). The increased attractiveness of the alternative might then produce a halo effect, in which component ratings are enhanced (Anderson, 1981).

Both dimensional weight and value shift models share in common the assumption that the decoy effects result from changes in processing of dimensional information, either by giving greater relative weight to a dimension or by altering the dimensional values of the alternatives. In their most general forms, it is unlikely that these two models can be distinguished from one another. However, the specific versions proposed here make different predictions about the effects of the three decoys shown in the middle panel. According to the dimensional weight model, the $R_{A}$ and $R F_{A}$ decoys should produce significant and equivalent shifts in choice preference through range extension, but the $F_{A}$ decoy should be ineffective. On the other hand, the value shift model predicts significant shifts of preference for all three decoys, with the $R F_{A}$ decoy producing the largest shifts because of the combined (additive) effects of range and frequency manipulations. Results of J. Huber et al. (1982) favor the dimensional weight model in that their frequency decoys did not produce significant shifts in choice preferences. However, Experiment 1 was designed to provide a more powerful test of these models.

\section{Dominance-Valuing Model}

An important thesis of modern cognitive theories of decision making is that the decision maker uses heuristic strategies that are adapted to features of the task and problem context (O. Huber, 1980; Kahneman \& Tversky, 1979; Payne, 1982; Payne, Bettman, \& Johnson, 1988; Svenson, 1979; Tversky \& Kahneman, 1981). Heuristic strategies tend to proceed dimensionwise rather than alternativewise and may not involve evaluation of all the information. Consistent with heuristic processing strategies, Russo and Dosher (1983) demonstrated that subjects in choice experiments tended to process 
information in a dimensionwise fashion across a variety of task environments.

Within a cost-benefit framework, the decision maker may prefer such strategies because they tend (a) to employ qualitative rather than more cognitively taxing quantitative comparisons, (b) to minimize the necessity to make difficult tradeoffs, (c) to provide a compelling and simple justification of the choice, and (d) to ensure minimally that poor alternatives will be avoided. Avoidance of poor alternatives seems particularly relevant to the kind of choice situation considered. The decoy is constructed so that it is dominated by at least one of the alternatives, and thus it is clearly a poor choice that should be avoided. The fact that a dominated alternative is rarely ever chosen (J. Huber et al., 1982; Ratneshwar et al., 1987; Simonson, 1989; Tyszka, 1983) suggests that subjects use a strategy that is sensitive to detecting dominance (see also Böckenholt, Aschenbrenner, Albert, \& Schmallhofer, in press). Any of a number of choice heuristics will be sensitive to dominance. Indeed, Montgomery (1980) has argued that the different heuristic strategies may be conceived as operators used in a search for a dominance structure among the choice alternatives.

Once the targeted alternative is perceived to dominate the decoy, subjects may choose it for any of several reasons. One possibility is that choice of the dominating alternative is more easily justified or explained (e.g., it dominates the decoy, but the other alternative does not; hence the targeted alternative is the superior choice). Simonson (1989) provided converging lines of evidence that are consistent with this hypothesis. First, subjects who anticipated having to publicly justify their choices showed significantly greater effects of the asymmetrically dominated decoy than those subjects who were assured that their responses would be kept totally confidential (although the decoy effect was not eliminated for these lowjustification subjects). Second, subjects in another study judged the choice of the dominating alternative to be significantly easier to justify and significantly less likely to be criticized than the choice of the nondominating alternative. These results suggest that asymmetrical dominance of a decoy enhances the overall attractiveness of the dominating alternative through the perception of greater justifiability of the choice.

The dominance-valuing model differs qualitatively from both the dimensional weight and value shift models in that although processing proceeds dimensionwise to detect dominance, the weighting of and values along the dimensions are assumed to remain fixed. Instead of a dimension-based process, choice preference for the targeted alternative is assumed to be altered by the perception of the dominance relationship, which directly increases the global attractiveness of the target. Because the dominance-valuing model proposes that the effects of the decoy are directly linked to the dominance relationship, this model's predictions for various positions of the decoy differ from the other two models. First, unlike the dimensional weight model, it predicts an effect of the $F_{A}$ decoy on choice preferences because this decoy lies in the region asymmetrically dominated by alternative $A$. Unlike the value shift model, the dominance-valuing model predicts equal effects of the three decoys $R_{A}, F_{A}$, and $R F_{A}$ on choice.
However, most important, it predicts no effects of the $R_{A}^{\prime}$ decoy shown in Figure 1, because this target is symmetrically dominated by both alternatives $A$ and $B$. In contrast, both dimensional weight and value shift models predict that the $R_{A}^{\prime}$ decoy should have effects on choice that are similar to those of the $R_{A}$ and $R F_{A}$ decoys, because all of these decoys asymmetrically extend the range along Dimension I but not along Dimension II. This prediction is tested in Experiments 2 and 3.

\section{Experiment 1}

As described above, a critical difference between the dimensional weight model and the other two models is that in their specified forms, only the dimensional weight model predicts that the $F$ decoy will fail to have an effect on choice behavior. This prediction was supported by the results of J. Huber et al. (1982), who reported significant effects of $R$ and $R F$ decoys on choice proportions but no significant effects of the $F$ decoys. However, conclusions that are based on a failure to reject the null hypothesis must always be considered within the context of the power of the test. The primary purpose of Experiment 1 was to provide a more powerful test of the asymmetrical dominance effect for the three types of decoys specified by range-frequency theory.

\section{Methods for Incrementing Power}

Several steps were taken to achieve a more powerful test. J. Huber et al. (1982) used stimuli that could be considered, for the most part, consumer services or products (e.g., different brands of beer, different film services). Although use of these stimuli facilitated generalization to the domain of consumer choice, it may have reduced the power of the test to detect preference reversals in two ways. First, subjects may be quite familiar with these types of stimuli and hence carry into the experiment a well-defined context, which would be expected to reduce the effects of the experimental manipulation of stimulus context (cf. Wedell, Parducci, \& Geiselman, 1987). Second, because these stimuli are quite memorable, preference reversals would be reduced by a tendency for subjects to simply choose the same alternative they remembered choosing previously. To avoid these problems in part, Experiment 1 used two-outcome gambles that differed in the probability to win and the amount to be won (the second outcome was always \$0). I assumed that the abstract nature of gambles would decrease the probability that subjects would be guided by preexisting contextual standards. Furthermore, because subjects made 20 choices from among 60 gambles during the course of the experiment, it seemed less likely that memory for specific choices would obscure preference reversals. ${ }^{2}$

\footnotetext{
${ }^{2}$ One of the six types of stimuli used by J. Huber, Payne, and Puto (1982) was a pair of two-outcome gambles, which resulted in no significant shifts in choice proportions. However, the strong and consistent effects on choice proportions reported in Experiments 1 and 2 of this article suggest that the lack of effect in their experiment was due to the particular pair of gambles used by those authors.
} 
A second method used to increase power was to increase the effect size. J. Huber et al. (1982) compared choice proportions under two conditions: (a) when a decoy favored one alternative and (b) when no decoy was present. In Experiment 1, each pair of gambles was presented once with the decoy favoring alternative $A$ and once with the decoy favoring alternative $B$, thus doubling the potential effect size. The exclusion of the no-decoy control from the present set of experiments precludes determination of the relative efficacy of different placement locations for the same type of decoy (i.e., whether range extension on the probability dimension has a greater effect than range extension on the money dimension, etc.). However, this control group was unnecessary to the central question of whether the various types of decoys differed in their relative efficacy in producing preference reversals.

A third step taken to increase power was to use a greater number of choice pairs and to combine these in statistical analyses. Thus, each subject made two choices for each of 10 pairs of target gambles. Choice proportions for each subject then approximated a continuous scale, so that more powerful parametric techniques could be used to analyze the data.

\section{Local Versus Global Context}

The procedure of Experiment 1 differed in one fundamental respect from procedures used by others in this area (J. Huber et al., 1982; Ratneshwar et al., 1987; Simonson, 1989; Tyszka, 1983): The local context, which is defined by the choice set, differed from the global context, which is defined by all of the stimuli in choice sets involving the same underlying dimensions for an experimental session. Previous researchers equated the local and global contexts by having subjects make only one choice for a given choice domain. In Experiment 1, each subject made choices for 20 sets of gambles, so that although local context was systematically manipulated by varying the particular decoy used for each set, the global contexts were roughly equated across choice sets.

The distinction between local and global contexts raises the broader issue of whether dimensional weight and value shift models should predict any effects of a within-subjects manipulation of which target the decoy favors. Range-frequency theory has generally assumed that the global context determines dimensional judgments (Parducci \& Wedell, 1986), which is consistent with the generally weak effects of sequential context (Ward \& Lockhead, 1970; however, for an example of strong effects of sequential context, see Parducci \& Sandusky, 1970). If dimensional weighting or valuation is based solely on the global context, there should be no effect of manipulating the local context across choice sets in Experiment 1 . Thus, to explain any context-induced preference reversals that may be observed in Experiment 1, both dimensional weight and value shift models must assume that the context strongly depends on the choice options in the current (local) set under consideration.

\section{Method}

Choice alternatives. The choice alternatives consisted of twooutcome gambles of the form $p$ to win $\$ X$ or else $1-p$ to win $\$ 0$, denoted here as $(p, \$ X)$. Figure 2 presents the five target gambles used in the study in a graphic form similar to that of Figure 1. Assuming a multiplicative integration of probability to win and amount to be won (Anderson \& Shanteau, 1970; Lopes, 1976; Shanteau, 1974), spacing along the two dimensions is determined by the logarithms of the values, so that the representation can be viewed additively. The preference vector depicts equal weight of $\log$ (probability to win) and $\log$ (amount to win), which corresponds to a model of preference that is based on the expected values of the gambles. The five target gambles had approximately the same expected value. Actual preferences were expected to correspond more closely to a model in which greater weight was given to $\log$ (probability to win) (Tversky et al., 1988).

Each target alternative was paired with each of the other four target alternatives twice, once with the decoy favoring it and once with the decoy favoring the alternative. Following the convention of Figure 1, the alternative in the pair with the higher value on Dimension II (i.e., probability to win) was designated as target $A$. Table 1 presents the decoy gambles, $R, F$, and $R F$, that were used for each target alternative when it occurred as target $A$ and when it occurred as target $B$. An important feature of the design of Experiment 1 was that for the middle three targets, the $R$ decoy used when the target was designated $A$ was the same as the $F$ decoy used when the target was designated $B$. For example, when $(.50, \$ 20)$ was paired with $(.40, \$ 25)$, the $R_{A}$ decoy was $(.50, \$ 18)$, extending the range downward on Dimension I. When $(.50, \$ 20)$ was paired with $(.67, \$ 15)$, it became the $B$ target; the same decoy gamble $(.50, \$ 18)$ was now an $F_{B}$ decoy, serving to increase the proportion of stimuli lying below target $B$ on Dimension I. Equating $R$ and $F$ decoys for the majority of targets provided a more direct test of the relative efficacy of these two types of decoys.

Booklets. Booklets consisted of a first page of instructions followed by four pages, each displaying five sets of choice triads. Instructions told subjects to imagine that they could play one of the three gambles in each set at no cost. Subjects were asked to circle the one gamble in each set that they would prefer to play.

Altogether, I constructed eight different stimulus pages. Four pages each consisted of one subset of five target pairs; the other four pages each consisted of the other subset of target pairs. I created two different arrangements of the target pairs on the page for each subset. The decoy for half of the pages favored one of the targets, but it favored the other target for the remaining pages.

From these pages, I constructed eight different booklets for each type of decoy under the following constraints. First, each of the 10

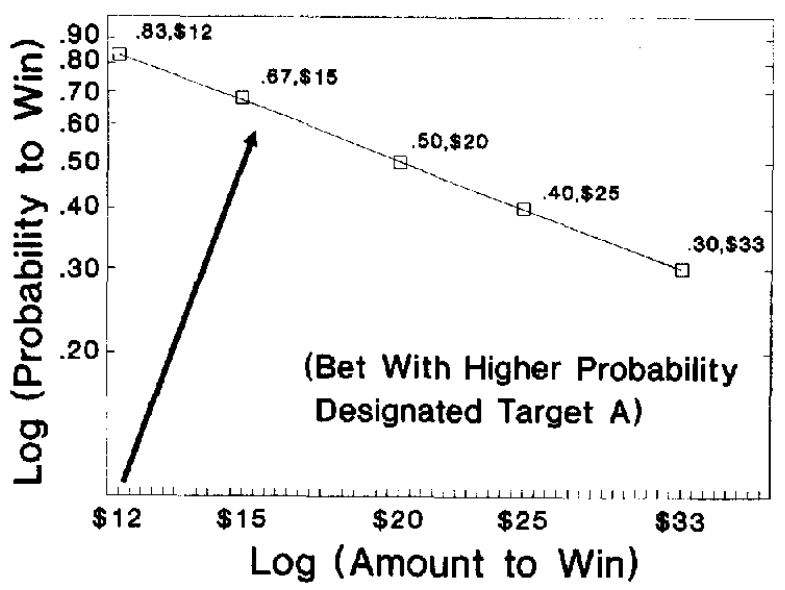

Figure 2. Target gambles of Experiments 1 and 2 mapped into a two-dimensional preference space (logarithmic spacing). 
Table 1

Gambles Used in Experiment 1

\begin{tabular}{cccc}
\hline \multicolumn{3}{c}{ Decoy bets } \\
\cline { 2 - 4 } Target bets & $R$ & $F$ & $R F$ \\
\hline Target $A$ & & & \\
$.40, \$ 25$ & $.40, \$ 20$ & $.35, \$ 25$ & $.35, \$ 20$ \\
$.50, \$ 20$ & $.50, \$ 18$ & $.45, \$ 20$ & $.45, \$ 18$ \\
$.67, \$ 15$ & $.67, \$ 13$ & $.62, \$ 15$ & $.62, \$ 13$ \\
$.83, \$ 12$ & $.83, \$ 10$ & $.78, \$ 12$ & $.78, \$ 10$ \\
Target $B$ & & & \\
$.30, \$ 33$ & $.25, \$ 33$ & $.30, \$ 30$ & $.25, \$ 30$ \\
$.40, \$ 25$ & $.35, \$ 25$ & $.40, \$ 20$ & $.35, \$ 20$ \\
$.50, \$ 20$ & $.45, \$ 20$ & $.50, \$ 18$ & $.45, \$ 18$ \\
$.67, \$ 15$ & $.62, \$ 15$ & $.67, \$ 13$ & $.62, \$ 13$ \\
\hline
\end{tabular}

Note. $R=$ range decoy; $F=$ frequency decoy; $R F=$ range-frequency decoy.

target pairs appeared once on page 2 or page 3 and then a second time on page 4 or page 5 , but with the decoy favoring a different alternative. Second, the targets favored on pages 2 and 3 were reversed for half of the booklets. Reversing which target in the set was initially favored for half of the booklets allowed for a test of whether the magnitude of the decoy effects was significantly reduced when the pair had previously been presented with the opposite decoy (i.e., transfer effects).

Subjects and design. The full design consisted of two withinsubjects variables, target pairs (10 pairs of bets) and context (favors $A$ or favors $B$ ), and two between-subjects variables, type of decoy $(R$, $F$, or $R F$ ) and order of presentation (8 orders) for a total of 24 between-subjects conditions. Eight University of Illinois undergraduates who received course credit for their participation were randomly assigned to each of the 24 between-subjects conditions for a total of 192 subjects. Of these, 1 subject was eliminated from the $F$ condition and 2 from the $R F$ condition for failing to complete the booklets. The dependent variable was the alternative chosen from each triad.

\section{Results and Discussion}

Figure 3 contains the results for the three types of decoys in contingency table form. In each contingency table, a subject is represented up to 10 times, corresponding to that subject's pattern of choices for the 10 pairs of target gambles. Choices for pairs of target gambles were excluded from the table whenever a decoy was chosen, which occurred for only $2 \%$ of the choice pairs. The strong tendency to avoid choosing the decoy is consistent with the assertion that subjects use choice strategies that guard against selection of a dominated alternative.

For each contingency table, the upper left and lower right cells represent consistent patterns of choice (i.e., choosing either $A$ or $B$ under both decoy conditions). The lower left and upper right cells represent preference reversals: The lower left cell represents the predicted pattern of choosing target $A$ when the decoy favors $A$ and choosing $B$ when the decoy favors $B$. The percentage of predicted preference reversals is substantial (20\%) and of similar magnitude for the three types of decoys. This pattern of results of closest to that predicted by the dominance-valuing model, according to which $R, F$, and $R F$ decoys aiter choice proportions to a similar degree. Furthermore, the occurrence of consistent decoy effects when global context is held constant means that if dimensional weight and value shift models are to explain the data, the context for determining weights or values must be closely tied to the specific choice sets (i.e., local contexts). Contextual theories that are based on global context effects cannot explain these data.

A repeated measures analysis of variance (ANOVA) was used to analyze the results, with the dependent variable corresponding to the arcsin transformation of the proportion of times subjects chose the higher probability bet (target $A){ }^{3}$ Subjects' responses were coded 1 if the subject preferred target $A, 0$ if the subject preferred target $B$, and .5 if the subject preferred the decoy. The dependent variable was calculated by taking the arcsin of the mean of the 10 responses under each contextual condition. There was no significant main effect for type of decoy, $F(2,186)=0.4, M S_{\mathrm{e}}=0.455, p>$ .50 , indicating that overall choice proportions did not differ for $R, F$, and $R F$ conditions. The effect of context was highly significant, $F(1,186)=52.8, M S_{\mathrm{e}}=0.067, p<.001$, reflecting the greater tendency to choose the alternative that was favored by the decoy. This effect was not contingent on the type of decoy used, as indicated by the nonsignificant Decoy $\times$ Context interaction term, $F(2,186)=1.8, M S_{\mathrm{e}}=0.067, p>.10$. Planned comparisons demonstrated strong effects of context for each of the three decoys $(p<.01)$.

The strong and significant effects of the $F$ decoy on choice are inconsistent with the dimensional weight model described earlier, in which the weight accorded a dimension is exclusively a function of the range of variation along that dimension. These effects are consistent with both value shift and dominance-valuing models. A difference between these latter two models is that the value shift model predicts greater effects for the $R F$ decoy because of additive effects of both range and frequency tendencies, but the dominance-valuing model predicts equivalent effects. A planned contrast comparing the effects of context for the $R F$ decoy with the average of the effects of context for the $R$ and $F$ decoys did not quite achieve significance, $F(1,186)=3.6, M S_{\mathrm{e}}=0.067, p=.06$. However, the direction of this effect is important to note: Contrary to the value shift model prediction, the $R F$ decoy showed reduced rather than enhanced effects of the contextual manipulation as compared with $R$ and $F$ decoys. Thus, there is clearly no support for an additive effect of the two types of decoys as implied by application of range-frequency theory.

J. Huber et al. (1982) found evidence for strong carryover effects as revealed by only a slight (3-point) shift in choice proportions for the within-subjects manipulation of context, as compared with a larger (9-point) difference for the betweensubjects manipulation. The fact that a large (14-point) shift was observed for the within-subjects manipulation in the present experiment indicates that asymmetrically dominated decoys can result in strong preference reversals. ${ }^{4}$ To examine

\footnotetext{
${ }^{3}$ The arcsin transformation was applied to reduce any systematic relationship between means and variances in the data. All analyses were run on untransformed proportions as well. Statistical conclusions that were based on the transformed and untransformed proportions did not differ.
} 


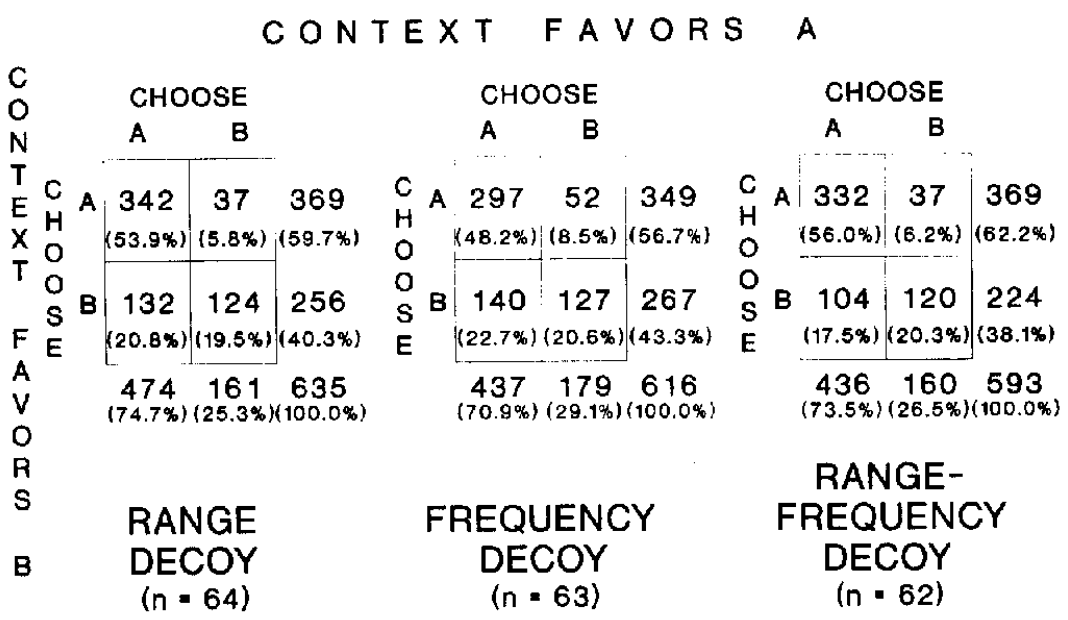

Figure 3. Contingency tables for choices in Experiment 1.

whether carryover effects occurred in the present experiment, an ANOVA was performed in which subjects' choice patterns under the two contextual conditions were examined for their first set of 10 choices versus their second set of 10 choices. A tendency to repeat prior choices when presented with the same target pair would result in reduced effects of context for the second set of choices as compared with the first. However, this was not the case. The choice proportions were nearly identical for the first and second sets, as reflected in the nonsignificant Order $\times$ Context interaction, $F(1,186)=0.1$, $M S_{\mathrm{e}}=0.085, p>.50$.

Finally, note that although the proportion of times the decoy was selected was low, it was not zero. Indeed, selection of the dominated decoy was greatest for the $R F$ decoy. To statistically test this effect, the proportion of times the decoy was chosen for each of the 10 pairs (in either context condition) was tallied for each subject. A one-way ANOVA was performed on the arcsin transformation of these proportions, with type of decoy as the between-subjects variable. Type of decoy significantly affected the proportion of times the decoy was chosen, $F(2,186)=5.0, M S_{\mathrm{e}}=0.772, p<.01$. NewmanKeuls post hoc comparison of the three pairs of means (at $p$ $<.05)$ showed that the selection of the dominated decoy was significantly greater for the $R F$ decoys than for either the $R$ or $F$ decoys but that the latter two did not significantly differ. One possible explanation of the greater selection of the decoy in the $R F$ condition is that unlike the $R$ and $F$ conditions, these decoys did not share any values in common with the targets (see Table 1). When triplets are presented simultaneously on a page, shared values can be detected at a glance. Shared values between a target and a decoy may then attract subjects' attention, so that they are more likely to directly compare these alternatives and hence detect dominance. When no values are shared (as with the $R F$ decoys), subjects may be less likely to generate the direct comparisons that enable them to detect (and value) dominance. According to the dominance-valuing model, failure to detect dominance should then lead to a reduction in the proportion of predicted reversals, which is again consistent with the data. Although this explanation is speculative, it could be tested in future process-tracing studies of the kind described by Russo and Dosher (1983): The prediction is that decoys that share values should be viewed earlier and more often in tandem with the target sharing the same value.

\section{Experiment 2}

The results of Experiment 1 demonstrated that the effects of an $F$ decoy on choice proportions can be strong and equivalent to those of the $R$ and $R F$ decoys. Although this pattern of effects is most consistent with the dominancevaluing model, the other two models could be modified to account for the pattern as well. Experiment 2 provided a more critical test between the dominance-valuing model and the other two models.

A shared assumption of dimensional weight and value shift models is that the effects of the decoy operate on a dimensionwise basis. Thus, the dimensional location of the decoy results in either a shift in the relative weights of the dimensions or shifts in the target values along the dimensions. The dominance-valuing model is fundamentally different from these two models in that perception of dominance itself increases the global value of the alternative rather than affecting dimensional values or weights. Thus, an important distinction between the two classes of models is that for the dominance-valuing model, asymmetrical dominance is a necessary and sufficient condition for decoy effects to occur, but this is not true for dimensional weight and value shift models.

This distinction implies that the $R_{A}^{\prime}$ decoy shown in the right panel of Figure 1 should provide a critical test between

\footnotetext{
${ }^{4}$ The 14-point difference reported may underestimate the magnitude of the decoy effect to some extent because a large number of subjects always chose the bet with the higher probability to win. This strategy does not allow for the possibility of preference reversals. If the analysis is restricted to the 87 subjects whose overall choice proportions for the higher probability bet were between .25 and .75 , a 21-point difference in choice proportions is observed. An ANOVA run for these subjects showed the same pattern of effects as for the full sample.
} 
the two classes of models. The $R^{\prime}{ }_{A}$ decoy has the same value along Dimension I as the $R_{A}$ and $R F_{A}$ decoys; hence it should alter either the dimensional weights or dimensional values in the same way as these two decoys according to the first two models. However, the $R^{\prime}{ }_{A}$ decoy differs from these other decoys in that it is dominated by both targets $A$ and $B$. According to dimensional weight and value shift models, the lack of asymmetrical dominance for the $R^{\prime}{ }_{A}$ decoy is irrelevant to the processes responsible for the decoy effects, and so there should be similar and significant effects of the $R^{\prime}{ }_{A}$ decoy on choice. According to the dominance-valuing model, the lack of asymmetrical dominance for the $R^{\prime}{ }_{A}$ decoy means no significant effects on choice should occur for this decoy.

\section{Method}

In nearly all respects, the method of Experiment 2 was identical to that of Experiment 1 . The only differences were that different decoys were used and subjects were selected from a different population.

Half of the booklets were a direct replication of the $R$ decoy condition of Experiment 1 . For the other half, the decoy was altered to correspond to the $R^{\prime}$ type decoy shown in Figure 1. This was done by simply altering the value that was unaltered for the $R$ decoy so that it matched the corresponding value of the nonfavored alternative. To illustrate this, consider the target pair $(.50, \$ 20)$ and $(.30, \$ 33)$. The $R_{A}$ decoy is $(.50, \$ 18)$, resulting from a manipulation of the dollar value of target $A$ (see Table 1 ). The $R^{\prime}{ }_{A}$ decoy $(.30, \$ 18$ ) was constructed by manipulating the dollar value in the same way but additionally changing the probability value so that it matched that of target $B$. In this way, the decoy was dominated by both targets, but the range was extended in a way that would only favor target $A$ (according to dimensional weight and value shift models).

Subjects were University of South Carolina undergraduates who received course credit for their participation. As in Experiment 1, 8 subjects were randomly assigned to each between-subjects conditions, for a total of 128 subjects. Data of 1 subject, who failed to complete the booklet, were dropped from the experiment.

\section{Results and Discussion}

Figure 4 contains the results for the two types of decoys in contingency table form. As in Experiment 1, a subject is

\begin{tabular}{|c|c|c|c|c|}
\hline \multirow{5}{*}{$\begin{array}{l}C \\
O \\
N \\
T \\
E \\
X \\
T\end{array}$} & \multicolumn{4}{|c|}{ CONTEXT } \\
\hline & \multirow{6}{*}{$\begin{array}{l}C_{H} \\
H \\
O \\
O \\
O \\
S \\
E\end{array}$} & \multicolumn{2}{|c|}{ CHOOSE } & \multirow{4}{*}{$\begin{array}{c}391 \\
(63.0 \%)\end{array}$} \\
\hline & & A & B & \\
\hline & & 358 & 33 & \\
\hline & & $\left(67.6 x_{)}\right)$ & (5.3\%) & \\
\hline & & 132 & 98 & 230 \\
\hline & & (21.3\%) & $(15.8 \%)$ & $(37.0 \%)$ \\
\hline & & $\begin{array}{c}490 \\
(78.9 \%)\end{array}$ & $\begin{array}{r}131 \\
(21.1 \%)\end{array}$ & $\begin{array}{c}621 \\
(100.0 \%)\end{array}$ \\
\hline & & RANGE & DEC & OY \\
\hline & & ASYMI & VET & \\
\hline & & DOMII & VAN & \\
\hline
\end{tabular}

$$
(n=64)
$$

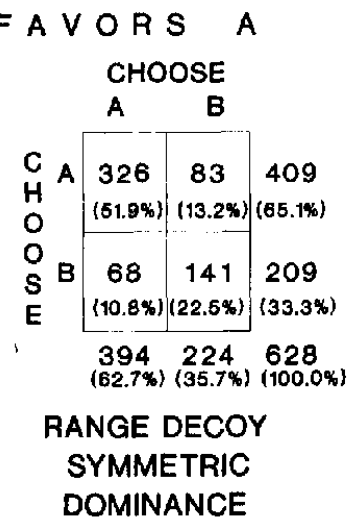$$
(n-63)
$$

Figure 4. Contingency tables for choices in Experiment 2. represented up to 10 times in each contingency table, corresponding to that subject's pattern of choices for the 10 pairs of target gambles. Choices for pairs of target gambles were excluded from the table whenever a decoy was chosen, which occurred for less than $2 \%$ of the choice pairs.

The results for the $R$ decoy were virtually the same as those presented in Figure 1; roughly $21 \%$ of the choice pairs reflected the predicted preference reversal, and only about $5 \%$ reflected the opposite tendency. However, the results for the $R^{\prime}$ decoy stood in stark contrast to this pattern: The percentage of predicted reversal was slightly smaller than the percentage of reversals in the opposite direction (11\% vs. $13 \%)$. The elimination of the predicted decoy effect when asymmetrical dominance was removed but asymmetrical range extension remained was consistent with the dominance-valuing model and inconsistent with dimensional weight and value shift models.

This pattern of results was statistically verified by repeated measures ANOVA on the arcsin of the choice proportions (calculated as in Experiment 1). The critical test is the Decoy $\times$ Context interaction, which was highly significant, $F(1,125)$ $=23.6, M S_{\mathrm{c}}=0.048, p<.001$. Planned comparisons were run (at $p<.05$ ) to test for the effects of context for each type of decoy separately. In accordance with all three models, context significantly altered choice proportions in the predicted direction for the $R$ decoy condition. However, contrary to both dimensional weight and value shift models, context did not significantly alter choice proportions for the $R^{\prime}$ decoy.

As in Experiment 1, a three-way ANOVA was run to assess order effects. Unlike Experiment 1, a significant Order $\times$ Context interaction was observed, $F(1,125)=4.3, M S_{\mathrm{e}}=$ $0.108, p<.05$, which reflected greater effects of context on the first set of judgments than on the second set. This interaction was not contingent on the type of decoy used $(F<$ 1.0). Although the presence of a carryover effect is inconsistent with Experiment 1, it is consistent with previous research (J. Huber et al., 1982) and the general expectation that subjects will repeat choices when faced with the same choice options.

\section{Experiment 3}

The results of Experiment 2 provide strong evidence that when a dominated decoy is included in the choice set, dominance must be asymmetrical for that decoy to affect choice. Experiment 1 also suggests that asymmetrical dominance is a sufficient condition for these effects to occur. However, the generality of the results of Experiments 1 and 2 may be challenged on two points. First, choices in these experiments involved abstract gambles. Thus, the extension of these results to more concrete choice options whose dimensions are likely to be additively, rather than multiplicatively, combined can be questioned.

Second, and more important, Experiments 1 and 2 manipulated the local context defined for each choice set rather than global context across choice sets. As discussed earlier, studies of context effects in judgment suggest that dimensional weight and value shift models should operate predominantly on the global context. Although models that were based on global context could not account for any of the effects of Experi- 
ments $I$ and 2, they may be applicable to situations in which global and local contexts are equated.

Experiment 3 was designed to address these two issues of generality. First, the three choice domains that showed the strongest decoy effects in the J. Huber et al. (1982) study were used: (a) cars that varied in rated gas mileage and rated ride quality, (b) restaurants that varied in quality rating and time to drive to the restaurant, and (c) television sets that varied in rated distortion of image and average years to break down. Second, each subject made only one choice for each of the three choice domains. By manipulating context between subjects for each domain, the local and global contexts were equated. As in Experiment 2, the $R$ and $R^{\prime}$ decoys were used, corresponding to range manipulations with asymmetrical dominance and symmetrical dominance, respectively. The dominance-valuing model, which asserts that asymmetrical dominance is necessary and sufficient for effects on choice proportions, predicts that choice proportions should differ strongly for $R_{A}$ versus $R_{B}$ decoys but should not differ for $R^{\prime}{ }_{A}$ versus $R^{\prime}{ }_{B}$ decoys. However, if dimensional effects as described by dimensional weight and value shift models operate when the local and global contexts are equated, then significant effects of the $R^{\prime}$ decoys on choice should be observed.

\section{Method}

Instructions, formatting of options, and actual values for the options were taken from the appendix provided by J. Huber et al. (1982). To maximize any effects of context, I used the "extreme range" manipulations described in their article. As in Experiment 2, the $R^{\prime}$ decoy was created by using the same range-altering value as the corresponding $R$ decoy but changing the other dimensional values to the corresponding value for the nonfavored alternative. Thus, for example, the $R_{A}$ decoy (100 ride quality [RQ], 21 miles per gallon [MPG]) favored target $A$ (100 RQ, $27 \mathrm{MPG)} \mathrm{over} \mathrm{target} B$ (80 RQ, $33 \mathrm{MPG}$ ) by extending the range downward on MPG. The $R^{\prime}{ }_{A}$ decoy (80 RQ, $21 \mathrm{MPG}$ ) similarly extended the range on the MPG dimension but matched target $B$ on the RQ dimension, so that dominance was symmetric.

The design consisted of the factorial combination of two betweensubjects variables, decoy $\left(R\right.$ or $\left.R^{\prime}\right)$ and context (favors $A$ or favors $B$ ), with targets $A$ and $B$ arbitrarily designated for each choice domain. Three counterbalanced arrangements of targets on the page were used to control for order effects. Ordering of the three choice sets was the same for all subjects: cars, then restaurants, then televisions. Between 29 and 34 subjects, sampled from the same population as in Experiment 2 , were randomly assigned to each of the four between-subjects conditions for a total of 127 subjects.

\section{Results and Discussion}

Table 2 contains the choice proportions for the three choice domains, segregated by type of decoy and contextual condition. These proportions exclude cases in which the decoy was chosen (which occurred only $3 \%$ of the time). When the decoy was asymmetrically dominated ( $R$ decoys), strong differences in choice proportions were observed between the two contexts in the predicted direction (averaging 41 points). However, when the decoy was symmetrically dominated ( $R^{\prime}$ decoys), the choice proportions were nearly identical under the two contextual conditions (the average difference being only 3 points). Thus, these results closely replicate those of Experiment 2.

A chi-square test of homogeneity of two proportions was conducted for each Decoy $\times$ Context condition in each choice domain. These results are shown in Table 2 . Only the asymmetrically dominated decoys produced statistically significant differences in choice proportions across contexts. As a more powerful test of these effects and to examine interaction effects, a two-way ANOVA was conducted with type of decoy ( $R$ or $R^{\prime}$ ) and context (favors $A$ or favors $B$ ) as betweensubjects factors. The dependent variable was the arcsin transformation of the proportion of choices favoring the $A$ target across choice domains (calculated as in Experiments 1 and 2). The main effect of context was highly significant, $F(1$, 123) $=14.3, M S_{\mathrm{e}}=0.197, p<.001$, but more important, so was the Decoy $\times$ Context interaction, $F(1,123)=14.2, M S_{\mathrm{e}}$ $=0.197, p<.001$. Planned comparisons showed that there were no significant effects of context for the $R^{\prime}$ decoys $(F<$ 1 ) but strong contextual effects for the $R$ decoys $(p<.001)$. Thus, the statistical tests support the conclusion that asymmetrical dominance is a necessary condition for these types of contextual effects on choice to occur.

\section{General Discussion}

Introducing an asymmetrically dominated decoy into the choice set increases the probability that the dominating alternative will be chosen. Although changes in dimensional weights or values could produce this effect, the results of Experiments 1-3 support an account that is more directly linked to the dominance structure of the choice alternatives. Experiment 1 demonstrated that asymmetrical dominance, whether produced by $R, F$ or $R F$ decoys, was a sufficient condition for producing preference reversals. Experiments 2

Table 2

Proportion Choosing Target $A$ in Experiment 3

\begin{tabular}{|c|c|c|c|c|c|c|}
\hline \multirow[b]{2}{*}{ Domain } & \multicolumn{3}{|c|}{ Asymmetric dominance } & \multicolumn{3}{|c|}{ Symmetric dominance } \\
\hline & $\begin{array}{c}\text { Context } \\
\text { favors } \mathrm{A}^{\mathrm{a}}\end{array}$ & $\begin{array}{l}\text { Context } \\
\text { favors } B^{b}\end{array}$ & Difference & $\begin{array}{c}\text { Context } \\
\text { favors } A^{c}\end{array}$ & $\begin{array}{l}\text { Context } \\
\text { favors B }\end{array}$ & Difference \\
\hline Automobiles & .688 & .188 & $.500^{*}$ & .310 & .346 & -.036 \\
\hline Restaurants & .424 & .061 & $.363^{*}$ & .355 & .241 & .114 \\
\hline TV sets & .938 & .559 & $.379^{*}$ & .774 & .769 & .005 \\
\hline
\end{tabular}

Note. Asymmetric dominance $=R$ decoy condition; symmetric dominance $=R^{\prime}$ decoy condition.

${ }^{2} n=33$. ' $n=34 . \quad{ }^{\mathrm{b}} n=31 . \quad{ }^{\circ} n=29$.

* Choice proportions differ significantly across contexts $(p<.001)$. 
and 3 underscored that when a dominated decoy is introduced, dominance must be asymmetrical to induce preference reversals. These latter two experiments provided no evidence for any additional dimensionwise effect of context on choice, regardless of whether context was manipulated locally for a given choice set or globally for all choice sets.

Although the dominance-valuing model was supported, these results did not address the specific mechanisms by which an alternative's dominance over the decoy increases its probability of being chosen. The data are consistent with Simonson's (1989) assertion that the dominating alternative is chosen because it is easier to justify. However, other heuristic strategies could produce the effect. Russo and Dosher (1983) reported that many of the information search patterns in their experiments were consistent with the majority of confirming dimensions (MCD) heuristic, in which subjects compared values of alternatives along each dimension and chose the alternative with the most "wins." J. Huber et al. (1982) noted that extension of the MCD strategy to three alternative choice sets (with the item with the most wins being chosen) could account for the asymmetrical dominance effect. Although Ratneshwar et al. (1987) found no evidence for the use of the MCD strategy (or any other standard heuristics) in subjects' retrospective protocols in this type of choice task, use of direct unobtrusive process-tracing techniques might be better suited for determining the extent to which heuristic strategies play a role in producing this type of context effect.

\section{Effects of Other Types of Decoys on Choice}

J. Huber and Puto (1983) demonstrated that introduction of nondominated decoys into a choice set can also produce large and disproportionate changes in the choice proportions for the remaining alternatives. These results have been replicated and extended by Ratneshwar et al. (1987) and Simonson (1989). Figure 5 contains three distinguishably different types of nondominated decoys. These decoys are located in the

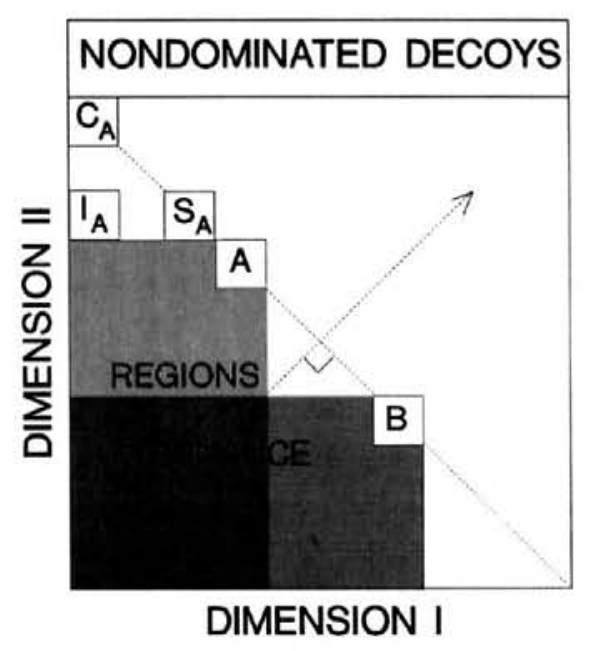

Figure 5. Nondominated decoys used in other studies: $I_{A}$ is inferior to $A, S_{A}$ is substitutable for $A$, and $C_{A}$ makes $A$ the compromise choice. triangular region that lies outside of the regions of dominance for Alternatives $A$ and $B$ and that is bounded by the equipreference contour they share.

Of the three, the decoy that is inferior in relation to $A$ (i.e., $I_{A}$ ) produces results that are closest to those produced by the asymmetrically dominated decoys explored in Experiments 1-3. This decoy increases the choice proportion for $A$, but it is rarely chosen (e.g., about $7 \%$ of choices in J. Huber \& Puto, 1983). One explanation for the similarity of the effects of the inferior and the asymmetrically dominated decoys is that subjects may effectively encode the $I_{A}$ decoy as asymmetrically dominated by $A$. Tversky (1969) provided evidence that small differences between stimuli along a dimension may be ignored or deemed unimportant by subjects, so that the stimuli are encoded as subjectively equivalent along that dimension. If the values of $I_{A}$ and $A$ in Figure 5 along Dimension II were encoded as equivalent, then $A$ would effectively dominate $I_{A}$, and hence the dimension-valuing model would apply.

The substitution $\left(S_{A}\right)$ and compromise $\left(C_{A}\right)$ decoys shown in Figure 5 result from moving the position of the $I_{A}$ decoy along either Dimension I or Dimension II toward the equipreference contour. These decoys are chosen a substantial percentage of the time (e.g., between $15 \%$ and $24 \%$ of choices in J. Huber \& Puto, 1983), but they have differing effects on selection of target $A$. The substitution decoy was introduced by Tversky (1972) in the context of his elimination by aspects (EBA) theory of choice. He demonstrated that including $S_{A}$ in the choice set reduced choices disproportionately more for $A$ than for $B$, violating independence from irrelevant alternatives (Luce, 1959). According to the EBA model, this effect results from subjects' processing information in a dimensionwise fashion and eliminating alternatives from the choice set that are below a threshold value on each successive dimension. Thus, subjects who process Dimension I first are more likely to eliminate $A$ and $S_{A}$ from the choice set and to choose $B$. Those who process Dimension II first are more likely to eliminate $B$ but to randomly choose between $A$ and its substitutable alternative $S_{A}$.

Like $S_{A}$, the $C_{A}$ decoy may lie on the same equi-preference contour as $A$, but its dimensional values differ considerably from those of $A$. Unlike the $S_{A}$ decoy, the effect of the $C_{A}$ decoy leads to a disproportionate increase in the proportion of choices of $A$ over $B$, and it can even lead to an increase in the absolute proportion choosing $A$ (J. Huber \& Puto, 1983; Simonson, 1989). Although the direction of this effect is the same as that for inferior and asymmetrically dominated decoys, it appears to result from a different process. First, unlike the latter two types of decoys, the $C_{A}$ decoy is chosen a substantial proportion of the time. Second, subjects' retrospective and on-line protocols commonly indicate that when the $C_{A}$ decoy was present, the $A$ decoy was judged attractive because it was perceived as a good compromise between the decoy and the other alternative (J. Huber \& Puto, 1983; Simonson, 1989).

One interpretation of the compromise effect is that contextual stimuli alter the location of ideal points in the psychological space. Coombs and Avrunin (1977) argue that singlepeaked preference functions result from a general approachavoidance conflict in choice in which the good attributes must 
be weighed against the bad attributes for each alternative. They assert that preference functions are generally single peaked because the utility of good attributes increases more slowly than the disutility of bad attributes. Consider then the advantage of selecting alternative $A$ from the set including $C_{A}$ and $B$. Although $C_{A}$ has a higher value than $A$ on Dimension II, it has a proportionately lower value than $A$ on Dimension $\mathrm{I}$, and because losses loom larger than gains, $A$ is preferred. Similarly, $B$ has a higher value than $A$ on Dimension I but a proportionately lower value on Dimension II, and again because the gain outweighs the loss, $A$ is preferred. Thus, the compromise effect is consistent with the relativistic weighing of gains and losses as described by ideal point theory.

Further evidence that contextual stimuli may shift ideal points and hence produce preference reversals is provided in a study by Riskey et al. (1979), in which subjects judged the pleasantness and sweetness of drinks with different concentrations of sugar. Because stimuli varied on only one dimension, asymmetrical dominance was not possible. When solutions were mostly very sweet (a negatively skewed distribution), a sweet solution (1.0 sucrose molarity) was judged as much more pleasant than was a less sweet solution $(0.11$ sucrose molarity), but the reverse was true for the positively skewed distribution. Judgments of sweetness showed the usual rangefrequency shifts. The data were consistent with a model of preference in which the ideal point is located at some moderate value along the relevant dimension (e.g., "slightly sweet") that shifts with context. Thus, these data suggest circumstances under which a value shift model may be applicable.

\section{Implications of Preference Reversals}

Preference reversals are important phenomena for several reasons. First, economists have been concerned with implications of the "irrational" behavior of failing to maintain a consistent preference ordering for rational models of choice in a market economy (cf. Grether \& Plott, 1979). However, as Machina (1987) pointed out, such violations of rational theory may be very restricted in scope, and other mechanisms (e.g., the tendency to repeat choices) may work to minimize their impact in the marketplace. Similarly, J. Huber and Puto (1983) argued that attraction and substitution effects may operate against one another, so that overall choice proportions appear consistent with independence from irrelevant alternatives.

Second, the point of view taken in this article is that preference reversals provide an opportunity to better understand the cognitive processes underlying judgments. That is, any theory of decision making under normal operations must also be able to account for the processes that produce the seemingly anomalous behavior of reversals and intransitivities of preference. In large part, much of the development of modern theories of the heuristics underlying decision and choice has been motivated and validated by studying decision anomalies (e.g., Kahneman \& Tversky, 1979; Payne, 1982; Svenson, 1979; Tversky \& Kahneman, 1981).

Third, Wedell (1990) pointed out how contextually induced reversals of preference ordering provide strong evidence for the early occurrence of contextual processes in the information-processing system. Most contextual effects in judgment produce only monotonic shifts of scale and so are subject to interpretation as rating-scale artifacts rather than substantive psychological phenomena (cf. Krantz, Luce, Suppes, \& Tversky, 1971, pp. 445-447). However, nonmonotonic effects on judgments or choices cannot be attributed to output processes. The present experiments indicate that dominance detection occurs early and has substantial psychological impact on the processes underlying choice.

\section{References}

Anderson, N. H. (1981). Foundations of information integration. San Diego, CA: Academic Press.

Anderson, N. H., \& Shanteau, J. C. (1970). Information integration in risky decision making. Journal of Experimental Psychology, 84, 441-451.

Birnbaum, M. H. (1974). Using contextual effects to derive psychophysical scales. Perception \& Psychophysics, 15, 89-96.

Birnbaum, M. H., \& Stegner, S. E. (1979). Source credibility in social judgment: Bias, expertise, and the judge's point of view. Journal of Personality and Social Psychology, 37, 48-74.

Böckenholt, U., Aschenbrenner, M., Albert, D., \& Schmallhofer, L. (in press). The effect of alternative dominance and attribute differences in information acquisition in multiattribute binary choice. Organizational Behavior and Human Decision Processes.

Braida, L. D., \& Durlach, N. I. (1972). Intensity perception: II. Resolution in one interval paradigms. Journal of the Acoustical Society of America, 51, 483-495.

Casey, J. T. (in press). Reversal of the preference reversal phenomenon. Organizational Behavior and Human Decision Processes.

Coombs, C. H., \& Avrunin, G. S. (1977). Single-peaked functions and the theory of preference. Psychological Review, 84, 216-230.

Eiser, J. R., \& Stroebe, W. (1972). Categorization and social judgment. San Diego, CA: Academic Press.

Fischhoff, B. (1983). Predicting frames. Journal of Experimental Psychology: Learning, Memory, and Cognition, 9, 103-116.

Fischhoff, B., Slovic, P., \& Lichtenstein, S. (1980). Knowing what you want: Measuring labile values. In T. Wallsten (Ed.), Cognitive processes in choice and decision behavior. Hillsdale, NJ: Erlbaum.

Fiske, S. T. (1980). Attention and weight in person perception: The impact of negative and extreme behavior. Journal of Personality and Social Psychology, 38, 889-906.

Goldstein, W. M., \& Einhorn, H. J. (1986). Expression theory and the preference reversal phenomena. Psychological Review, 94, 236254.

Gravetter, F., \& Lockhead, G. R. (1973). Criterial range as a frame of reference for stimulus judgment. Psychological Review, 80, 203216.

Grether, D. M., \& Plott, C. R. (1979). Economic theory and the preference reversal phenomenon. American Economic Review, 69, 623-638.

Helson, H. (1964). Adaptation-level theory. New York: Harper \& Row.

Hershey, J. C., \& Schoemaker, P. J. H. (1980). Prospect theory's reflection hypothesis: A critical examination. Organizational Behavior and Human Performance, 25, 395-418.

Huber, J., Payne, J. W., \& Puto, C. (1982). Adding asymmetrically dominated alternatives: Violations of regularity and the similarity hypothesis. Journal of Consumer Research, 9, 90-98.

Huber, J., \& Puto, C. (1983). Market boundaries and product choice: 
Illustrating attraction and substitution effects. Journal of Consumer Research, 10, 31-44.

Huber, O. (1980). The influence of some task variables on cognitive operations in an information-processing decision model. Acta Psychologica, 45, 187-196.

Kahneman, D., \& Tversky, A. (1979). Prospect theory: An analysis of decisions under risk. Econometrica, 47, 263-291.

Kahneman, D., \& Tversky, A. (1984). Choices, values, and frames. American Psychologist, 39, 341-350.

Krantz, D. H., Luce, R. D., Suppes, P., \& Tversky, A. (1971). Foundations of measurement. San Diego, CA: Academic Press.

Lichtenstein, S., \& Slovic, P. (1971). Reversal of preferences between bids and choices in gambling decisions. Journal of Experimental Psychology, 89, 46-55.

Lichtenstein, S., \& Slovic, P. (1973). Response-induced reversal of preference in gambling: An extended replication in Las Vegas. Journal of Experimental Psychology, 101, 16-20.

Lindman, H. R. (1971). Inconsistent preference among gambles. Journal of Experimental Psychology, 89, 390-397.

Lopes, L. L. (1976). Model-based decision and inference in stud poker. Journal of Experimental Psychology: General, 105, 217239.

Luce, R. D. (1959). Individual choice behavior: A theoretical analysis. New York: Wiley.

Machina, M. J. (1987). Decision-making in the presence of risk. Science, 236, 537-543.

Mellers, B. A. (1982). Equity judgments: A revision of Aristotelian views. Journal of Experimental Psychology: General, 111, 242-270.

Montgomery, H. (1980). Decision rules and the search for a dominance structure: Towards a process model of decision making. In P. Humphreys, O. Svenson, \& A. Vari (Eds.), Analysing and aiding decision processes (pp. 343-369). Amsterdam: North-Holland.

Parducci, A. (1965). Category judgment: A range-frequency model. Psychological Monographs, 77 (2, Whole No. 565).

Parducci, A. (1983). Category ratings and the relational character of judgment. In H.-G. Geissler \& V. Sarris (Eds.), Modern trends in perception (pp. 89-105). East Berlin, German Democratic Republic: Deutscher Verlag der Wissenschaften.

Parducci, A., \& Perrett, L. F. (1971). Category rating scales: Effects of relative spacing and frequency of stimulus variables. Journal of Experimental Psychology Monograph, 89, 427-452.

Parducci, A., \& Sandusky, A. (1970). Limits on the applicability of signal detection theory. Perception \& Psychophysics, 7, 63-64.

Parducci, A., \& Wedell, D. H. (1986). The category effect with rating scales: Number of categories, number of stimuli, and method of presentation. Journal of Experimental Psychology: Human Perception and Performance, 12, 496-516.

Payne, J. W. (1982). Contingent decision behavior. Psychological Bulletin, 92, 382-402.

Payne, J. W., Bettman, J. R., \& Johnson, E. J. (1988). Adaptive strategy selection in decision making. Journal of Experimental Psychology: Learning, Memory, and Cognition, 14, 534-552.

Ratneshwar, S., Shocker, A. D., \& Stewart, D. W. (1987). Toward understanding the attraction effect: The implications of product stimulus meaningfulness and familiarity. Journal of Consumer Research, 13, 520-533.

Riskey, D. R., Parducci, A., \& Beauchamp, G. K. (1979). Effects of context in judgments of sweetness and pleasantness. Perception \& Psychophysics, 26, 171-176.

Russo, J. E., \& Dosher, B. A. (1983). Strategies for multiattribute binary choice. Journal of Experimental Psychology: Learning, Memory, and Cognition, 9, 676-696.

Schneider, S. L., \& Lopes, L. L. (1986). Reflection in preferences under risk: Who and when may suggest why. Journal of Experimental Psychology: Human Perception and Performance, 12, 535548.

Shanteau, J. P. (1974). Component processes in risky decision making. Journal of Experimental Psychology, 103, 680-691.

Simonson, I. (1989). Choice based on reasons: The case of attraction and compromise effects. Journal of Consumer Research, 16, 158174.

Skowronski, J. J., \& Carlston, D. E. (1989). Negativity and extremity biases in impression formation: A review of explanations. Psychological Bulletin, 105, 131-142.

Slovic, P., Fischhoff, B., \& Lichtenstein, S. (1982). Response mode, framing, and information processing effects in risk assessment. In R. Hogarth (Ed.), New directions for methodology of social and behavioral science: Question framing and response consistency. (Vol. 11, pp. 21-36). San Francisco: Jossey-Bass.

Slovic, P., \& Lichtenstein, S. (1983). Preference reversals: A broader perspective. American Economic Review, 73, 596-605.

Svenson, O. (1979). Process descriptions of decisions. Organizational Behavior and Human Performance, 23, 86-112.

Tversky, A. (1969). Intransitivity of preferences. Psychological Review, 76, 31-48.

Tversky, A. (1972). Elimination by aspects: A theory of choice. Psychological Review, 79, 281-299.

Tversky, A., \& Kahneman, D. (1981). The framing of decision and the psychology of choice. Science, 211, 453-458.

Tversky, A., Sattath, S., \& Slovic, P. (1988). Contingent weighting in judgment and choice. Psychological Review, 95, 371-384.

Tyszka, T. (1983). Contextual multiattribute decision rules. In Sjöberg (Ed.), Human decision making (pp. 243-256). Amsterdam: North-Holland.

Ward, L. M., \& Lockhead, G. R. (1970). Sequential effects and memory in category judgments. Journal of Experimental Psychology, 84, 27-34.

Wedell, D. H., (1990). Methods for determining the locus of context effects in judgment. In J. P. Caverni, J. M. Fabre, \& M. Gonzalez (Eds.), Cognitive biases (pp. 285-302). Amsterdam: North-Holland.

Wedell, D. H., \& Böckenholt, U. (1990). Moderation of preference reversals in the long run. Journal of Experimental Psychology: Human Perception and Performance, 16, 429-438.

Wedell, D. H., Parducci, A., \& Geiselman, R. E. (1987). A formal analysis of ratings of physical attractiveness: Successive contrast and simultaneous assimilation. Journal of Experimental Social Psychology, 23, 230-249.

Wedell, D. H., Parducci, A., \& Lane, M. (1990). Reducing the effects of the immediate context in clinical judgment: Effects of number of categories and type of anchors. Joumal of Personality and Social Psychology, 16, 429-438.

Wedell, D. H., Parducci, A., \& Roman, D. (1989). Student perceptions of fair grading: A range-frequency analysis. American Journal of Psychology, 102, 233-248.

Received July 30, 1990

Revision received December 20, 1990 Accepted December 27, 1990 